\title{
High-precision measurement of the proton's atomic mass
}

\author{
F. Heiße, ${ }^{1,2}$ F. Köhler-Langes, ${ }^{1}$ S. Rau,${ }^{1}$ J. Hou,${ }^{1}$ S. Junck,${ }^{3}$ A. Kracke, ${ }^{1}$ \\ A. Mooser ${ }^{4}$ W. Quint, ${ }^{2}$ S. Ulmer, ${ }^{4}$ G. Werth,${ }^{3}$ K. Blaum, ${ }^{1}$ and S. Sturm ${ }^{1}$ \\ ${ }^{1}$ Max-Planck-Institut für Kernphysik, Saupfercheckweg 1, 69117 Heidelberg, Germany \\ ${ }^{2}$ GSI Helmholtzzentrum für Schwerionenforschung GmbH, Planckstraße 1, 64291 Darmstadt, Germany \\ ${ }^{3}$ Institut für Physik, Johannes Gutenberg-Universität, 55099 Mainz, Germany \\ ${ }^{4}$ RIKEN, Ulmer Fundamental Symmetries Laboratory, Wako, Saitama 351-0198, Japan
}

(Dated: June 22, 2017)

\begin{abstract}
We report on the precise measurement of the atomic mass of a single proton with a purpose-built Penning-trap system. With a precision of 32 parts-per-trillion our result not only improves on the current CODATA literature value by a factor of three, but also disagrees with it at a level of about 3 standard deviations.
\end{abstract}

The properties of the basic building blocks of matter shape a network of fundamental parameters, which are crucial to develop precise quantitative understanding of nature and its symmetries. One of these fundamental constants is the mass of the proton $m_{p}$, which has always been a target and yardstick of precision experiments 1 5]. It is thus correlated with most other parameters of atomic physics. For example, its value influences the Rydberg constant [6], and it is also required for the precise comparison of the masses of the proton and antiproton, in order to perform a stringent test of CPT invariance via a hydrogen anion [7.

All recent proton mass values are based on Penningtrap measurements, where the cyclotron frequencies $\nu_{c}=\frac{1}{2 \pi} \frac{q}{m} B$ of the proton (or $\mathrm{H}_{2}^{+}$) and a reference ion with respective charge-to-mass ratios $q / m$ are compared in the same magnetic field $B$. In this letter we report on a high-precision measurement of $m_{p}$ in atomic mass units, which is based on cyclotron frequency comparisons of protons and highly charged carbon $\left({ }^{12} \mathrm{C}^{6+}\right)$ ions. While the largely different charge-to-mass ratio between the proton and the ${ }^{12} \mathrm{C}^{6+}$ ion imposes technical challenges to be discussed later, the comparison with the atomic mass standard allows us to determine the mass of the proton directly in atomic mass units. In order to do so, we have to relate the mass of the ${ }^{12} \mathrm{C}^{6+}$ ion to that of a ${ }^{12} \mathrm{C}$ atom:

$$
m\left({ }^{12} \mathrm{C}^{6+}\right)=m\left({ }^{12} \mathrm{C}\right)-6 m\left(e^{-}\right)+\sum_{i=1}^{6} \frac{E_{b, i}}{c^{2}} .
$$

Here, $E_{b, i}$ denotes the binding energies of the six removed electrons, $c$ the speed of light in vacuum and $m_{e}$ the electron mass [8]. Since $m\left({ }^{12} \mathrm{C}\right)$ is $12 \mathrm{u}$ by definition and the atomic mass of the electron has been previously determined by our group with $2.9 \times 10^{-11}$ relative uncertainty, this relation is limited only by the knowledge of electronic binding energies. The currently tabulated values in the NIST table of ionization energies [9] allow to derive $m\left({ }^{12} \mathrm{C}^{6+}\right)=11.9967096264139(10) \mathrm{u}$ with a relative precision of 0.08 parts-per-trillion (ppt), which does not pose any limitation on the precision of the proton's atomic mass reported here.

The measurements have been carried out in a highlyoptimized, purpose-built cryogenic Penning-trap setup, dedicated to mass measurements on light ions, which is a successor experiment of the Mainz $g$-factor experiment for highly charged ions [10 12]. While the superconducting magnet and the experiment's liquid helium cryostat have been re-used, both the trap section as well as the cryogenic electronics and detection circuitry have been newly developed. This was necessary to adress the specifically strong requirements on the quality of the trapping fields, set by the low mass and charge of the proton and resulting large motional amplitudes.

The highly charged ${ }^{12} \mathrm{C}^{6+}$ ion as well as the proton are created in the hermetically sealed and cryogenically cooled trap chamber using an integrated miniature electron beam ion source (EBIS) [13, 14, which ablates atoms from a carbon nanotube-filled PEEK target (TECAPEEK [15]). After creation, the ions are shuttled to the measurement trap (MT, see Fig. 1) by adiabatically shifting the electrostatic trapping potential along the magnetic field axis. Here, all ions except for one proton or one ${ }^{12} \mathrm{C}^{6+}$ ion, respectively, are ejected from the trap. After that, the ion of interest is transported to a "storage" trap (ST-I, see Fig. 1). Next, a new cloud of ions is produced in the EBIS and the process is repeated to place the second ion in the MT. Storing both ions of interest simultaneously in the same trap setup but at different locations allows rapid swapping of the ions between the MT and one of the two STs, and thus drastically reduces the time between measurements of the two ions [7. Additionally, the creation process can change the effective electric potential by charging unavoidable nonconductive patches on the trap electrodes also referred to as patch potentials, which is also avoided by our method since no reloading is required. Cryopumping in the sealed chamber provides a virtually perfect vacuum of better than $10^{-17}$ mbar, which prevents any unwanted interaction of the ion of interest and enables storage times of the ions in excess of months. In principle this would allow to perform the complete measurement with only one single 


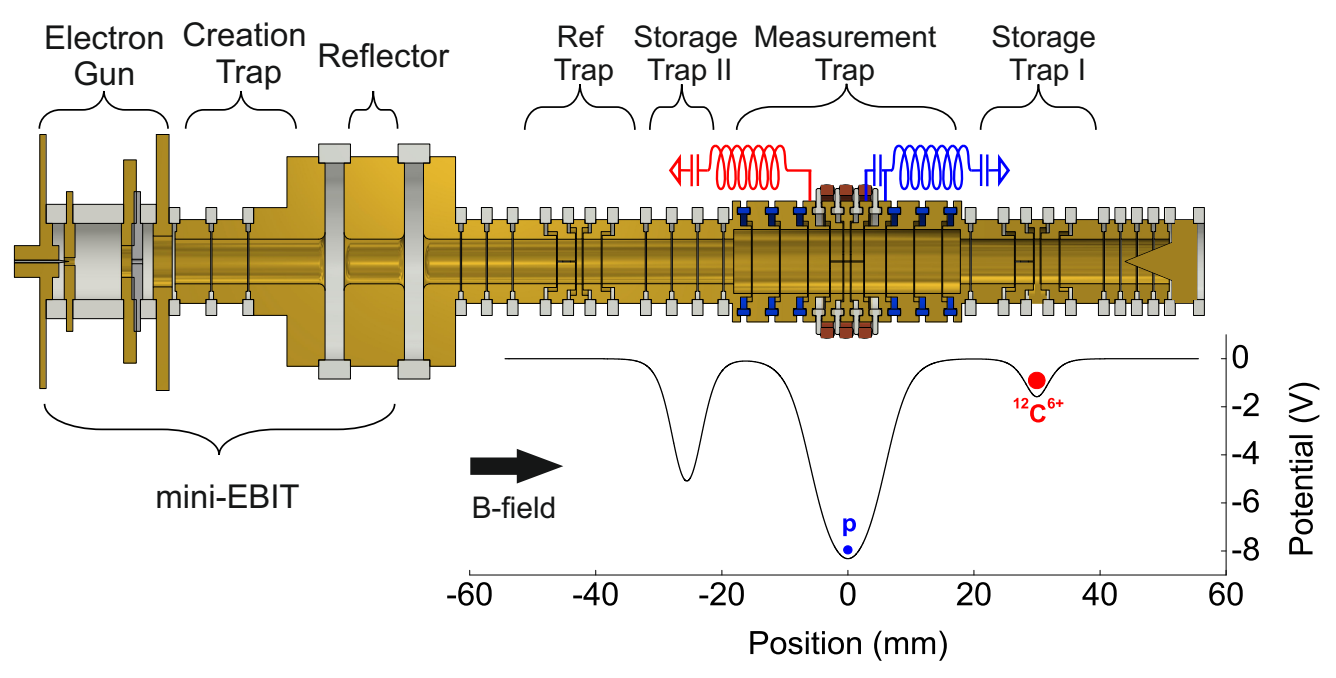

FIG. 1. Sketch of the trap setup. The trap tower includes two separate storage traps (ST-I, ST-II), the measurement trap (MT) and a reference trap (RT) for magnetic field monitoring, which is presently not used. Ions are created in-situ using a mini-EBIT [13. By shuttling the ions between the storage traps and the MT, the time between successive measurements is minimized. Individual superconducting detection circuits for the proton (blue) and for the carbon ion (red), allow measurements at the identical electrostatic field configurations and thus guarantee the identical position and magnetic field. For details see text.

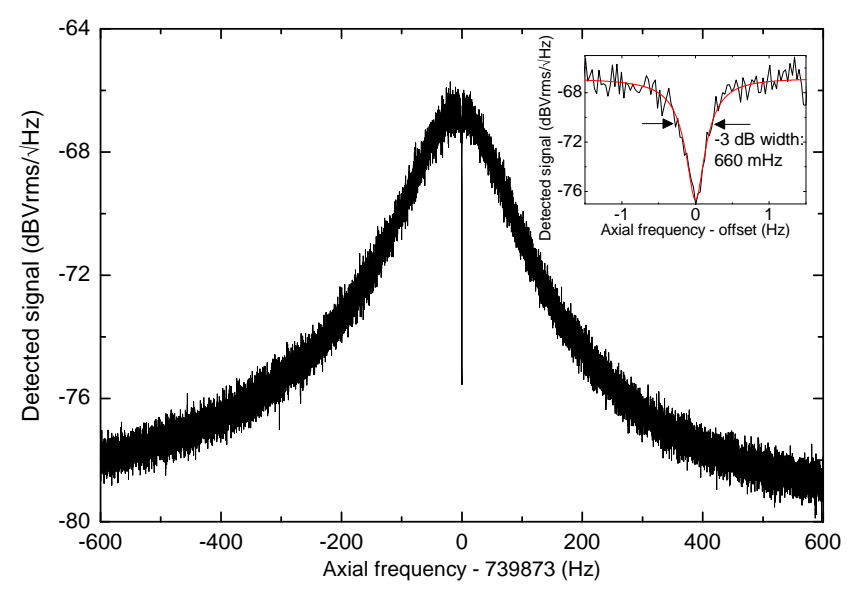

FIG. 2. Illustration of a typical dip spectrum for the determination of the proton axial frequency. The inset shows a zoom to the dip signal, together with our fitted lineshape model. For details see text.

pair of ions. Only to exclude systematic effects arising from a possible residual contamination of the trap with other ions, we repeated the measurement with two newly created pairs of ions.

Of the three independent Penning-trap eigenmotions, the tank circuit can only detect the axial motion directly [16. In order to determine the cyclotron frequency of the stored ions, we measure the image current the ions induce on the trap electrodes when oscillating with the axial eigenfrequency $\nu_{z}$ of about $525 \mathrm{kHz}$ for ${ }^{12} \mathrm{C}^{6+}$ and $740 \mathrm{kHz}$ for the proton, respectively. This tiny current is transformed into a measurable voltage by a highlysensitive superconducting tank circuit, which is picked up by a low-noise cryogenic amplifier [17. Especially the proton with its low charge requires a high quality factor ( $Q$-value) of the detection circuit to produce a sufficient signal. By operating the trap and its electronics at cryogenic temperature $(4 \mathrm{~K})$, the temperature of the tank circuit and with it also the kinetic energy of the ions is reduced, which strongly suppresses systematic effects and increases precision. Still, the low mass $m_{p}$ of the proton translates into relatively large motional amplitudes for a given temperature, asking for exquisitely well-defined electromagnetic trapping fields. Additionally, the finite kinetic energy of the ion during the measurement causes a relativistic mass increase, which is specifically strong for the light proton. Finally, even in a shimmed superconducting magnet, the field is not perfectly homogeneous. In order to guarantee that both ions are measured at exactly the same location, it is thus important to use the identical trap voltage configuration for both ions. The large charge-to-mass ratio mismatch of the two ions, and the respective large voltages, would lead to shifts of the equilibrium position. These shifts are unavoidable and hard to control due to patch potentials. Combined with the residual inhomogeneity of the magnetic field this would cause a systematic error in the measured cyclotron frequency ratio. However, since it is currently technically impossible to tune a high- $Q$ tank circuit over the required frequency range, we have instead implemented for the first time two independent tank circuits, fine-tuned to the exact ratio of the axial frequencies with a voltagevariable capacitor. In this way we can keep the exact 
same voltage setting for the measurement with respect to the two ions. The axial frequency can be determined from a fit to the noise dip that appears as a unique signature of the ion when it is in thermal equilibrium with the tank circuit (see Fig. 2). This dip has a $3 \mathrm{~dB}$-width of $660 \mathrm{mHz}$ for the proton and $1100 \mathrm{mHz}$ for the carbon ion, respectively. After an averaging time of three minutes, a fit allows to determine the axial frequency with a precision of about $50 \mathrm{mHz}$. The other two frequencies, the modified cyclotron frequency $\nu_{+}$and the magnetron frequency $\nu_{-}$, have to be determined by coupling them to the axial motion with radio frequency drives on the motional sidebands [18]. When driving the ion at the "red" axial-cyclotron sideband at $\nu_{+}-\nu_{z}$, the axial motion is dressed with the cyclotron state, leading to a splitting of the dip signal into two dips ("double-dip"), from which the cyclotron frequency can be determined. For the determination of the magnetron frequency a similar technique is applied. From the three eigenfrequencies the free cyclotron frequency can be calculated via the invariance relation $\nu_{c}=\sqrt{\nu_{+}^{2}+\nu_{z}^{2}+\nu_{-}^{2}}$ [19], where $\nu_{+} \approx 57 \mathrm{MHz}$ for the proton and $\nu_{+} \approx 29 \mathrm{MHz}$ for the carbon ion, respectively. Since the modified cyclotron frequency dominates this relation, the relative precision of its determination is of highest importance. For this reason, we utilize the phase-sensitive PnA technique [20, which allows determining $\nu_{+}$with highest precision and very low kinetic energy of the ion, and thus low systematic frequency shifts. Furthermore, the influence of temporal magnetic field fluctuations is reduced compared to the double-dip technique, which requires longer measurement times. Both the PnA and the double-dip technique are employed during the measurement campaign. While the PnA technique is about an order of magnitude more precise for a single measurement than the doubledip method, the comparison of the two techniques allows for an important internal consistency check. Moreover, the PnA technique is less prone to systematic shifts due to any imperfection in the lineshape.

The sequence of a single measurement is illustrated in Fig. 3. At the beginning of each single ratio measurement, a random generator selects the ion that gets measured first. This cancels the effect of a possible systematic linear drift of the magnetic field. The first ion is then transported to the MT and the other ion is shifted into its associated storage trap. In both cases, the voltage in all three traps is set to the same values, such that the electrostatic potential is truly identical. Then the doubledip measurement of $\nu_{+}$is performed, followed by an axial frequency measurement. Subsequently, the modified cyclotron frequency is measured again, this time with the PnA technique. Directly following the last PnA cycle, the ions are swapped and the second half of the measurement cycle starts with a $\mathrm{PnA}$ measurement on ion II, such that the time in between the cyclotron frequency

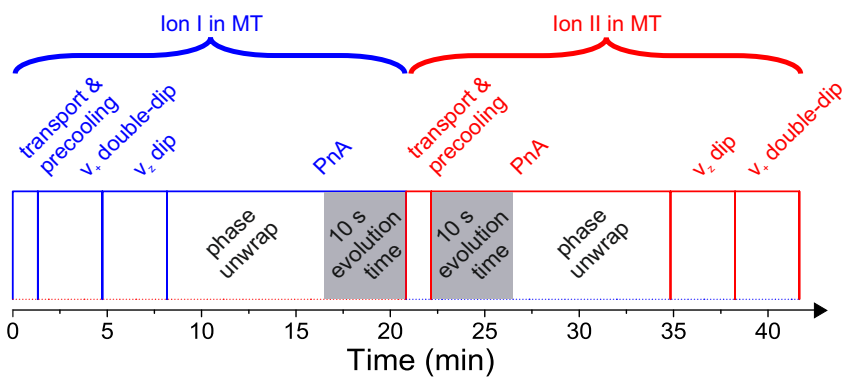

FIG. 3. Illustration of the measurement sequence. In the beginning of each step, ion I is chosen randomly to exclude linear magnetic field drifts and systematic shifts arising from the measurement procedure. Ion I is then transported into the MT, and ion II into the respective storage trap. In any case, both storage traps are set to their nominal voltage to prevent systematic influence on the equilibrium position of the measured ion in the MT. After cooling the cyclotron motion as well as the axial motion, $\nu_{+}$and $\nu_{z}$ are measured with the dip methods, respectively. Ten PnA cycles at different phase evolution times: six times $10 \mathrm{~ms}, 0.1 \mathrm{~s}, 1 \mathrm{~s}, 2 \mathrm{~s}$ and $5 \mathrm{~s}$ ensure the precise determination of the initial phase and a proper phase unwrapping 20. Then four cycles of the PnA method are applied, each with $10 \mathrm{~s}$ phase evolution time to determine $\nu_{+}$with highest precision. Finally ion I is moved away and ion II is loaded into the MT and its frequencies are measured in reverse order. Each such cycle gives an individual value for the mass ratio.

measurements of the two ions is minimized. Finally, also for the second ion the axial and cyclotron frequencies are measured using the double-dip and single dip method, respectively. During one 43-minute measurement cycle the cyclotron frequency ratio is determined with a relative uncertainty of $1.8 \times 10^{-10}$.

The major systematic shift arises from the finite kinetic energy of the ions. The thermal distribution of axial mode energies, which are thermalized with the tank circuit, leads to a motion within the residual inhomogeneity of the magnetic field. Owing to the axial symmetry of the motion, only even orders of field moments are relevant, with the quadratic "magnetic bottle" $\left(B_{2} / B_{0}=-7.2(4) \times 10^{-8} / \mathrm{mm}^{2}\right)$ component being the leading order contribution. The resulting shift of the modified cyclotron frequency is particularly large for the proton, due to its low charge, and a factor of six smaller for the ${ }^{12} \mathrm{C}^{6+}$ ion. To reduce the size of this shift, the axial temperature of the ions is reduced below the ambient cryogenic temperature to about $T=1.7(1.0) \mathrm{K}$ by means of electronic feedback cooling [21, which limits this shift to $\delta \nu_{c} / \nu_{c}=-44(28)$ ppt for the proton. In order to determine this temperature we first perform sideband coupling of the cyclotron and the axial modes. Then a burst excitation of the cyclotron motion maps the initial axial amplitude distribution to the axial frequency via the residual magnetic bottle $B_{2}$.

Apart from the axial temperature also the cyclotron 
TABLE I. Systematic shifts and their uncertainties for the individual cyclotron frequencies and their ratio $R_{0}$. For details see text. The second column gives the specific relative systematic shifts for the proton (left) and carbon (right) cyclotron frequencies, respectively. These values apply for the smallest modified cyclotron radius of the proton $(9 \mu \mathrm{m})$ and the carbon ion $(14 \mu \mathrm{m})$ during the phase evolution time of PnA. The values in column three and four denote the relative systematic shifts and their uncertainties for $R_{0}$ after the extrapolation to zero cyclotron energy for both ions. The lineshape model shift only occurs for the first two ion pairs due to a slight offset between the ion's and the detector's resonance frequencies and is therefore not included in the total. The magnetron frequency is not measured in each cycle, but due to its small value also the resulting uncertainties of $\nu_{c}$ and $R_{0}$ are negligible within the given limits.

\begin{tabular}{lccc}
\hline \hline Effect & Rel. syst. shift of $\nu_{c}\left(10^{-11}\right)$ & Rel. syst. shift of $R_{0}\left(10^{-11}\right)$ & Uncertainty $\left(10^{-11}\right)$ \\
$r_{+}^{\text {exc }}$ for $\mathrm{p} /{ }^{12} \mathrm{C}^{6+}(\mu \mathrm{m})$ & $9 / 14$ & $0 / 0$ & $0 / 0$ \\
\hline Image charge & $0.83 / 9.94$ & 9.10 & 0.46 \\
Image current & $-0.14 /-0.33$ & -0.19 & 0.03 \\
Residual magnetostatic inhomogeneity & $4.43 / 0.14$ & -3.95 & 2.75 \\
Residual electrostatic anharmonicity & $\ll 0.01 / \ll 0.01$ & -1.14 & $\ll 0.01$ \\
Special relativity & $7.23 / 3.45$ & 0.27 & 0.71 \\
Lineshape mode|a & $-0.03 / 0.14$ & 0 & 0.30 \\
Magnetron frequency uncertainty & $0.01 / 0.06$ & 3.82 & 0.06 \\
\hline Total & $12.33 / 13.40$ & 2.89 \\
\hline \hline
\end{tabular}

a The typical value varies slightly between measurement sets due to different detunings of the axial resonators.

energy after the excitation within the PnA cycle contributes to the systematic shifts. Here, the mass increase due to special relativity is dominant and mainly affecting the proton, resulting in a shift of $\delta \nu_{c} / \nu_{c}=-72(8) \mathrm{ppt}$ for the proton. By an extrapolation using varying excitation amplitudes it can be corrected for (see Fig. 4). The absence of any non-statistical jitter in the corrected ratios gives us great confidence in the validity of our systematics model.

Additionally to the above-mentioned energy dependent effects, the major systematic shift arises from the interaction of the ion with the trap electrodes. Due to the axial symmetry of the trap, the ion's image charges in the electrodes mainly produce an outward force on the ion in the radial direction. This shifts both the modified cyclotron frequency and the magnetron frequency, but leaves the axial frequency unaffected apart from a tiny contribution due to the slits between the trap electrodes. For that reason, the shift does not cancel out when using the invariance relation of the three motional frequencies and has to be calculated and corrected. However, compared to previous measurements [8], our new and larger trap helps to diminish this effect, which scales as

$$
\frac{\delta \nu_{c}}{\nu_{c}}=-C_{\mathrm{IC}} \frac{m}{8 \pi \epsilon_{0} r_{0}^{3} B_{0}^{2}},
$$

where $r_{0}=5 \mathrm{~mm}$ denotes the trap radius and $B_{0}=3.76 \mathrm{~T}$. The coefficient $C_{\mathrm{IC}}$ depends on the exact dimensions of the electrodes and their gaps and has been determined numerically to $C_{\mathrm{IC}}=1.97(10)[22$. Due to the dependence on the ion's mass, this shift affects mostly the carbon ion, where it amounts to $\delta \nu_{c} / \nu_{c}=-99 \mathrm{ppt}$. The uncertainty of this shift is given by the manufactur- ing precision of the trap electrodes of $\pm 10 \mu \mathrm{m}$, allowing to correct the shift to better than 5 ppt. Apart from the immediate interaction of the ion with its image charges, depending on the impedances of the electronics attached to the electrodes, also the resulting image current interacts with the ion. Close to their respective resonances, high- $Q$ tank circuits boost this interaction and can lead to sizeable frequency pulling 23 . To control and reduce this effect, our cyclotron tank circuits, which are used for faster identification of the ions, are detuned by several line-widths during the measurement. The residual shift is estimated to $\delta \nu_{c} / \nu_{c}=3.3(2)$ ppt for the carbon ion and even smaller for the proton. The same effect in the axial motion is taken care of by our lineshape model, which corrects for the frequency pulling. However, due to imperfect knowledge of the resonator parameters and a slight off-resonance of the ions in some of our measurements this contributes to systematic uncertainties. Our new seven-electrode cylindrical trap enables the adjustment of the electrostatic potential in a way that contributions to the systematic error budget are completely negligible. This allows us to parametrically amplify the ion motion at the end of the PnA cycle to large enough amplitudes for achieving a sufficient signal to apply PnA to the proton, which is challenging due to its low charge. A summary of all shifts is listed in Table[1. From our measurements and the extrapolation to zero cyclotron energy we obtain (see Fig. 4):

$$
\left.R_{0} \equiv \frac{\nu_{c}\left({ }^{12} \mathrm{C}^{6+}\right)}{\nu_{c}(p)}\right|_{\text {stat }}=0.5037763676431(77)
$$

Applying all corrections according to Table I we arrive 


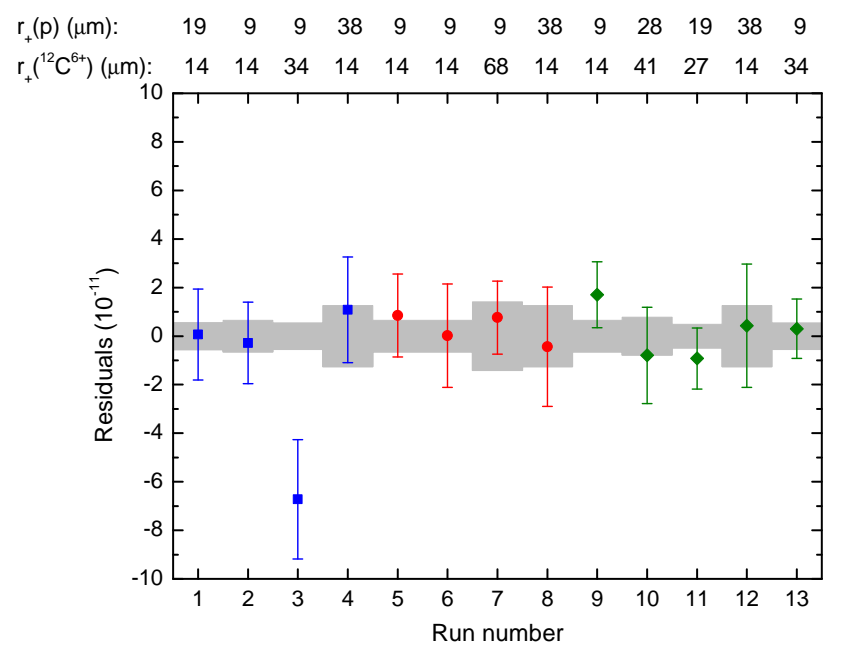

FIG. 4. Residuals of the 3-parameter $\left(R_{0}, a, b\right)$ fit with $R_{i}=R_{0}+a\left(A_{t, i}(p)\right)^{2}+b\left(A_{t, i}\left({ }^{12} \mathrm{C}^{6+}\right)\right)^{2}$ to the measured frequency ratios $R_{i}$. Here, $A_{t, i}(p)$ and $A_{t, i}\left({ }^{12} \mathrm{C}^{6+}\right)$ denote the effective dipole excitation strength, the product of excitation amplitude and time for the modified cyclotron motion of the proton and the carbon ion, respectively. The shown modified cyclotron radii can be calculated via $r_{+, i}=\kappa \times A_{t, i}$, where the parameter $\kappa$ is extracted from $a$ and $b$, respectively. This is checked via an independent calibration of the amplitudes by means of a frequency shift due to the residual magnetic inhomogeneity and shows good agreement. The grey area indicates the prediction interval of the fit, the error bars indicate the statistical uncertainty of the individual measurement. The agreement for the complete range of modified cyclotron energies indicates the validity of our model of systematics. The data set consists of three separate ion pairs, indicated by the color of the data points. The agreement of the data sets renders an influence of parasitically trapped ions or electrons improbable.

at:

$$
\left.R_{\text {final }}\right|_{\text {stat,sys }}=0.5037763676624(77)(146) .
$$

By correcting for the mass of the missing electrons and their respective binding energies in the ${ }^{12} \mathrm{C}^{6+}$ ion, taken from [9], we can relate the mass of the carbon ion to the atomic mass unit as shown in Eq. (1). Finally, using $m_{p}=R_{\text {final }} m\left({ }^{12} \mathrm{C}^{6+}\right) / 6$ we calculate the proton mass in atomic mass units:

$$
m_{p}=1.007276466583(15)(29) \mathrm{u} .
$$

Here, the two numbers given in brackets are the statistical and systematic uncertainties of the measurement, respectively. Thus, our value of $m_{p}$ has a relative precision of $32 \mathrm{ppt}$, which is three times more precise than the current CODATA value (Fig. 50 [6] but shows a deviation from the literature value by more than three standard deviations.

As an additional cross-check we can use the double-dip

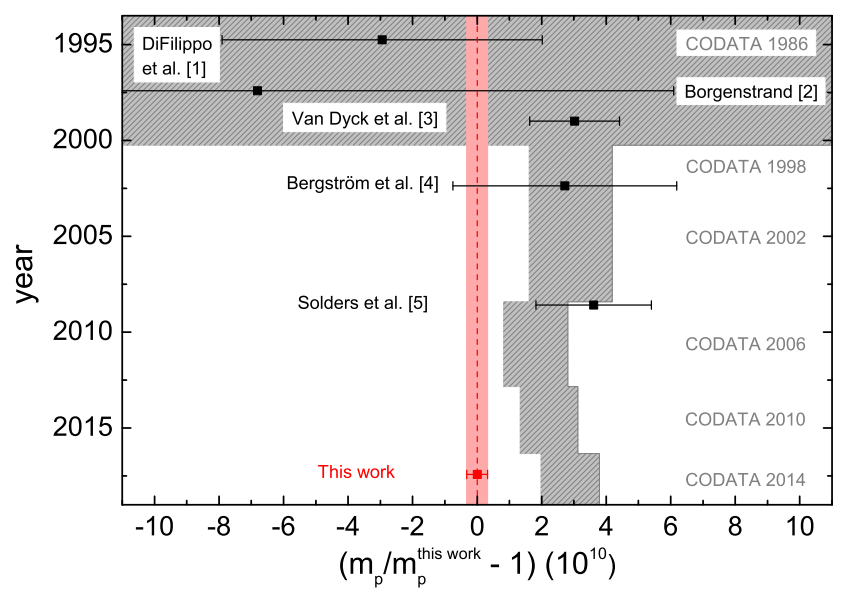

FIG. 5. Comparison of our result to previous values for the proton's atomic mass. Mainly two Penning-trap experiment's contribute to the literature value, the UW-PTMS at the University of Washington [3] and the SMILETRAP spectrometer in Stockholm [2, 4, 5]. Our value disagrees with the latest CODATA value at a level of 3.3 standard deviations.

measurements of $\nu_{+}$instead of the PnA, which yields:

$$
\left.R_{\mathrm{DD}}\right|_{\text {stat,sys }}=0.50377636766(3)(5) .
$$

The result from the double-dip data is in excellent agreement with the PnA result, however by a factor of around four less precise. To further confirm the measured $3.3 \sigma$ deviation we conducted a sequence of cross-check mass measurements. To this end, we have performed similar measurements as above, however replacing the highly charged carbon ion by ${ }^{16} \mathrm{O}^{8+}$. Using the respective version of Eq. (1) and associated ionization energies [9] for ${ }^{16} \mathrm{O}$ and our new value for $m_{p}$ we obtain

$$
m\left({ }^{16} \mathrm{O}\right)=15.99491461924(54)(43)(53) \mathrm{u},
$$

where the last bracket contains the uncertainty arising from our measured proton mass. The relative uncertainty of this result is $5.4 \times 10^{-11}$, and with an $0.4 \sigma$ deviation it is in excellent agreement with the literature value of the AME2016 $m\left({ }^{16} \mathrm{O}\right)=15.99491461960(17) \mathrm{u}$ 24. Finally, we performed a comparison of the cyclotron frequency ratio of ${ }^{12} \mathrm{C}^{3+}$ and ${ }^{12} \mathrm{C}^{6+}$. The measured mass agrees to the calculated one within $0.5 \sigma$ with an uncertainty of $1.1 \times 10^{-10}$, where the relative systematic uncertainty is only $7 \times 10^{-12}$.

Using our result, the proton-electron-mass ratio can be determined with a relative precision of $43 \mathrm{ppt}$, where the uncertainty arises nearly equally from the proton and the electron mass. This is a factor of two more precise compared to the current value [6]:

$$
m_{p} / m_{\mathrm{e}}=1836.152673346(81) .
$$

The shifted proton mass also impacts the ${ }^{3} \mathrm{He}$ "mass puzzle" [25, 26], which indicated a possible inconsistency 
of the existing determination of the mass of the $\mathrm{HD}^{+}$ molecule compared to ${ }^{3} \mathrm{He}^{+}$. The inconsistency of $4 \sigma$ is reduced by a factor of around two using our measurement result. By applying our measurement scheme also with the deuteron, we will be able to further address the ${ }^{3} \mathrm{He}^{+}$ inconsistency. Furthermore, $m_{p}$ affects the atomic mass of the neutron [26], but results in a shift of smaller than $1 \sigma$, due to the dominant uncertainty in the deuteron's binding energy. The influence on the Rydberg constant $R_{\infty}$ [27] is currently small, since its error is dominated by the charge radius of the proton. However, the more precise value for $R_{\infty}$ that could be extracted from the muonic hydrogen experiment once the proton radius puzzle [28. can be resolved will be significantly influenced by our result.

In summary we performed the most precise measurement of the atomic mass of the proton. Our measurement is a factor of three more precise compared to the current literature value, however shifted by about three standard deviations. In a set of carefully conducted cross-check measurements we have confirmed a series of other literature values and were not able to track any yet uncovered systematic effects imposed by our method. Combined with the independently measured electron mass this measurement yields a factor of two more precise proton-electron-mass ratio, too.

The main systematic limitation of our measurement is given by the residual quadratic magnetic field component combined with the finite axial motion amplitude of the ions. In the next phase of our experiment we plan to significantly improve on this limitation by compensating the first and second order magnetic inhomogeneities with a dedicated set of in-situ superconducting magnetic shims. Additionally, common-mode magnetic field fluctuations will be canceled by simultaneous phase-sensitive measurements in the RT and MT, allowing for significantly longer measurement times and correspondingly a lower statistical uncertainty.

\section{ACKNOWLEDGEMENTS}

We want to thank Marc Schuh for the calculation of the image charge shift in our trap. This work was supported by the Max Planck Society, the EU (ERC grant no 290870; MEFUCO), the International Max Planck Research School for Quantum Dynamics in Physics, Chemistry and Biology (IMPRS-QD) and the RIKEN FPR Funding as well as the RIKEN Incentive Research Project Program.
[1] F. DiFilippo, V. Natarajan, K. R. Boyce, and D. E. Pritchard, Phys. Rev. Lett. 73, 1481 (1994)

[2] H. Borgenstrand, An Attempt to Measure the Proton Mass Using a Penning Trap and Highly-Charged Ions, Ph.D. thesis, Stockholm University (1997).

[3] R. S. Van Dyck Jr., D. L. Farnham, S. L. Zafonte, and P. B. Schwinberg, AIP Conf. Proc. 457, 101 (1999)

[4] I. Bergström, T. Fritioff, R. Schuch, and J. Schönfelder, Phys. Scr. 66, 201 (2002).

[5] A. Solders, I. Bergström, S. Nagy, M. Suhonen, and R. Schuch, Phys. Rev. A. 78, 2514 (2008).

[6] P. J. Mohr, D. B. Newell, and B. N. Taylor, Rev. Mod. Phys. 88, 035009 (2016).

[7] S. Ulmer, C. Smorra, A. Mooser, K. Franke, H. Nagahama, G. Schneider, T. Higuchi, S. Van Gorp, K. Blaum, Y. Matsuda, et al., Nature 524, 196 (2015)

[8] S. Sturm, F. Köhler, J. Zatorski, A. Wagner, Z. Harman, G. Werth, W. Quint, C. H. Keitel, and K. Blaum, Nature 506, $467(2014)$

[9] http://physics.nist.gov/asd [2017, May 17], A. Kramida, Yu Ralchenko, J. Reader and the NIST ASD Team.

[10] S. Sturm, A. Wagner, B. Schabinger, J. Zatorski, Z. Harman, W. Quint, G. Werth, C. H. Keitel, and K. Blaum, Phys. Rev. Lett. 107, 023002 (2011)

[11] A. Wagner, S. Sturm, F. Köhler, D. A. Glazov, A. V. Volotka, G. Plunien, W. Quint, G. Werth, V. M. Shabaev, and K. Blaum, Phys. Rev. Lett. 110, 033003 (2013)

[12] F. Köhler et al., Nat. Commun. 7, 10246 (2016)

[13] B. Schabinger, J. Alonso, K. Blaum, G. Werth, H.-J. Kluge, W. Quint, M. Vogel, and S. Stahl, J. Phys. Conf. Ser. 58, 121 (2007)

[14] S. Sturm, K. Blaum, B. Schabinger, A. Wagner, W. Quint, and G. Werth, J. Phys. B 43, 074016 (2010)

[15] http://www.ensinger-online.com/de [2017, April 30], Ensinger GmbH, Nufringen, Germany.

[16] L. S. Brown and G. Gabrielse, Rev. Mod. Phys. 58, 233 (1986).

[17] H. Nagahama, G. Schneider, A. Mooser, C. Smorra, S. Sellner, J. Harrington, T. Higuchi, M. Borchert, T. Tanaka, M. Besirli, et al., Rev. Sci. Instrum. 87, 113305 (2016)

[18] E. A. Cornell, R. M. Weisskoff, K. R. Boyce, and D. E. Pritchard, Phys. Rev. A 41, 312 (1990)

[19] L. S. Brown and G. Gabrielse, Phys. Rev. A 25, 2423 (1982)

[20] S. Sturm, A. Wagner, B. Schabinger, and K. Blaum, Phys. Rev. Lett. 107, 143003 (2011)

[21] B. D'Urso, B. Odom, and G. Gabrielse, Phys. Rev. Lett. 90, 043001 (2003).

[22] M. Schuh, (private communications).

[23] V. Natarjan, Penning Trap Mass Spectroscopy at 0.1 $p p b$, Ph.D. thesis, Massachussetts Institute Of Technology (1993).

[24] W. Huang, G. Audi, M. Wang, F. Kondev, S. Naimi, and X. Xu, Chin. Phys. C 41, 030002 (2017)

[25] E. G. Myers, A. Wagner, H. Kracke, and B. A. Wesson, Phys. Rev. Lett. 114, 013003 (2015)

[26] S. L. Zafonte and R. S. Van Dyck Jr., Metrologia 52, 280 (2015).

[27] S. G. Karshenboim and V. G. Ivanov, Appl. Phys. B 123, 18 (2016)

[28] R. Pohl et al., Nature 466, 213 (2010). 